\title{
Integrative genomic analyses of WNT11: Transcriptional mechanisms based on canonical WNT signals and GATA transcription factors
}

\author{
MASUKO KATOH $^{1}$ and MASARU KATOH ${ }^{2}$ \\ ${ }^{1}$ M\&M Medical BioInformatics, Hongo 113-0033; ${ }^{2}$ Genetics and Cell Biology Section, \\ National Cancer Center, Tokyo 104-0045, Japan
}

Received April 22, 2009; Accepted May 29, 2009

DOI: 10.3892/ijmm_00000227

\begin{abstract}
We and others previously cloned and characterized vertebrate WNT11 orthologs, which are involved in gastrulation, neurulation, cardiogenesis, nephrogenesis, and chondrogenesis during fetal development. WNT11 orthologs activate both canonical and non-canonical WNT signaling cascades depending on the expression profile of WNT receptors, such as Frizzled family members, LRP6, ROR2, and RYK. Human WNT11 is expressed in breast cancer, gastric cancer, esophageal cancer, colorectal cancer, neuroblastoma, Ewing sarcoma, and prostate cancer. Canonical WNT signals and GATA family members are involved in WNT11 transcription during embryogenesis of model animals; however, precise mechanisms of WNT11 expression remain unclear. Here, refined integrative genomic analyses of WNT11 are carried out to elucidate the mechanisms of WNT11 transcription. The WNT11 gene was found to encode two isoforms by using alternative first exons. WNT11 isoform A (NM_004626.2 RefSeq) consists of exons 2, 3, 4, 5 and 6, whereas WNT11 isoform B consists of exons $1,2,3,4,5$ and 6 . We identified double TCF/LEFbinding sites within the proximal promoter regions (-48-bp position from the TSS of human WNT11 isoform B and -43-bp position from the TSS of human WNT11 isoform A), and also double GATA-binding sites within intron 2 of human WNT11 gene (+933-bp and +5001-bp positions from TSS of human WNT11 isoform A). Double TCF/LEF- and double GATAbinding sites within the regulatory regions of human WNT11 gene were conserved in other mammalian WNT11 orthologs. These facts indicate that canonical WNT signals and GATA family members directly upregulate WNT11 transcription. Canonical WNT-induced WNT11 activates non-canonical WNT signaling cascades to induce cellular movement, and also
\end{abstract}

Correspondence to: Dr Masaru Katoh, Genetics and Cell Biology Section, National Cancer Center, 5-1-1 Tsukiji, Chuo Ward, Tokyo 104-0045, Japan

E-mail: mkatoh-kkr@umin.ac.jp

Key words: WNT, gastrulation, neurulation, migration, mesenchymal stem cells, chondrogenesis, gastric cancer, peritoneal dissemination, bioinformatics, personalized medicine activates the $\mathrm{Ca}^{2+}$-MAP3K7-NLK signaling cascade to break the canonical WNT signaling. Canonical WNT-to-WNT11 signaling loop is involved in cellular migration during embryogenesis as well as tumor invasion during carcinogenesis.

\section{Introduction}

WNT family members are secreted proteins with glycolipid modifications, which are involved in embryogenesis, adulttissue homeostasis, and carcinogenesis (1-5). WNT1, WNT2, WNT2B, WNT3, WNT3A, WNT4, WNT5A, WNT5B, WNT6, WNT7A, WNT7B, WNT8A, WNT8B, WNT9A, WNT9B, WNT1OA, WNT10B, WNT11, and WNT16 genes are conserved in the mammalian genomes (6), whereas additional wnt family genes other than the conserved 19 genes exist in the nonmammalian vertebrate genomes.

There is growing evidence that WNT signals are transduced to the canonical and non-canonical WNT signaling cascades in a context-dependent manner (7-10). LRP5, LRP6, and Frizzled family members are involved in the canonical WNT signaling cascade to activate the transcription of target genes based on the $\beta$-catenin-TCF/LEF complex, whereas ROR1, ROR2, RYK, and Frizzled family members are involved in the non-canonical WNT signaling cascades, including DVL-RhoA-ROCK, DVL-RhoB-Rab4, DVL-RacJNK, DVL-aPKC, $\mathrm{Ca}^{2+}$-Calcineurin-NFAT, $\mathrm{Ca}^{2+}$-MAP3K7NLK, $\mathrm{Ca}^{2+}-\mathrm{MAP} 3 \mathrm{~K} 7-\mathrm{NF}-\kappa \mathrm{B}$, and DAG-PKC signaling cascades (1-5,11-17).

We and other groups have cloned and characterized vertebrate WNT11 orthologs (18-23). WNT11-related gene, wnt11r, exists in the zebrafish, Xenopus, and chicken genomes, but not in the mammalian genomes (24-26). WNT11 orthologs activate both canonical and non-canonical WNT signaling cascades depending on the expression profile of WNT receptors, such as Frizzled family members, LRP6, and RYK (27-34). Vertebrate WNT11 homologs are involved in fetal development, especially in gastrulation $(23,35-37)$, neural crest migration $(26,38)$, cardiogenesis (39-43), nephrogenesis $(44,45)$, and chondrogenesis $(46,47)$.

WNT11 is expressed in several types of human cancer. We reported expression of human WNT11 in breast cancer, gastric cancer, esophageal cancer, and embryonal tumor $(1,19)$. We also reported upregulation of WNT11 in some cases 

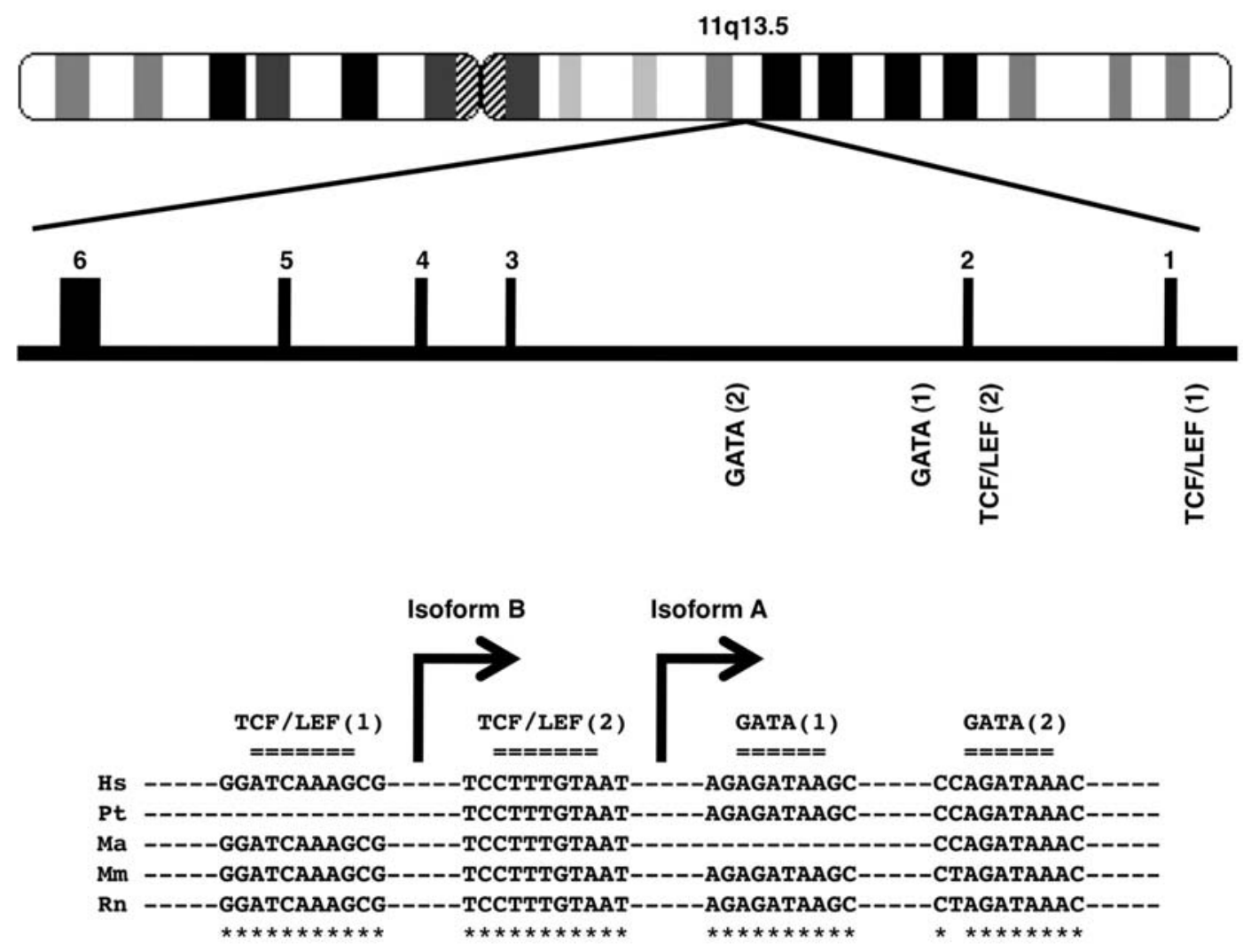

Figure 1. Integrative genomic analyses of WNT11. Schematic representation of WNT11 gene at human chromosome $11 \mathrm{q} 13.5$ is shown in the upper part. WNT11 gene encodes two splicing variants by using alternative first exons. Conserved transcription factor-binding sites within WNT11 regulatory regions are shown in the lower part. Hs, human; Pt, chimpanzee; Ma, macaque; Mm, mouse; Rn, rat.

of primary colorectal cancer (19). Other groups then reported expression of human WNT11 in neuroblastoma (48), Ewing sarcoma (49), and prostate cancer (50). Because precise mechanisms of WNT11 expression remain unclear, refined integrative genomic analyses of WNT11 were carried out to elucidate the mechanisms of WNT11 transcription.

\section{Materials and methods}

Comparative genomic analyses. Human genome sequences corresponding to human WNT11 RefSeq (NM_004626.2) were searched for by using BLAST programs, as previously described $(51,52)$. WNT11 expressed sequence tags (ESTs) were also searched for to identify WNT11 splicing variants, and also to determine the putative transcription start site (TSS) $(53,54)$. Conserved transcription factor-binding sites within WNT11 promoters were then searched for based on manual inspection, as previously described $(55,56)$.

Regulatory network analyses. The literature on WNT, Hedgehog, and Notch signaling molecules and GATA family transcription factors in PubMed and Medline databases was critically evaluated to extract knowledge on the regulation of TCF/ LEF, GLI, FOX, CSL, and GATA transcription factors. The mechanisms of WNT11 transcription were then investigated based on our data of conserved transcription factor-binding sites within WNT11 promoters and in-house knowledgebase of transcription factors regulated by the stemcell signaling network.

\section{Results}

WNT11 splicing variants transcribed by using alternative promoters. BLAST programs using NM_004626.2 RefSeq as a query sequence revealed that human WNT11 gene is located within human genome sequence AP000785.4, as previously described (57). BLAST programs using the WNT11 genome sequence as a query sequence next revealed that 11 ESTs are transcribed from the same first exon of human WNT11 gene as NM_004626.2 RefSeq, and that three ESTs are transcribed from alternative first exon located about 4-kb upstream position compared with NM_004626.2 RefSeq. DA812463.1, AW009544.1, and AW294719.1 ESTs transcribed from the alternative first exon are spliced to the first exon of NM_004626.2 RefSeq. To distinguish two alternative first exons of human WNT11 gene, the first exon of NM_004626.2 RefSeq was renamed exon 2, and the alternative first exon was designated exon 1. WNT11 isoform A (NM_004626.2 RefSeq) consists of exons 2, 3, 4, 5 and 6, whereas WNT11 isoform $\mathrm{B}$ consists of exons 1, 2, 3, 4, 5 and 6 (Fig. 1).

Comparative genomics on mammalian WNT11 orthologs. Chimpanzee WNT11 gene, macaque WNT11 gene, mouse Wnt 11 gene, and rat Wnt11 gene are located within NW_001222304.1, NW_001100387.1, AC093351.8 and NW_047561.1 genome sequences, respectively. Comparative genomic analyses of mammalian WNT11 orthologs revealed that the WNT11 promoter B region located at the 5'-adjacent position of exon 1, the WNT11 promoter A region located at 


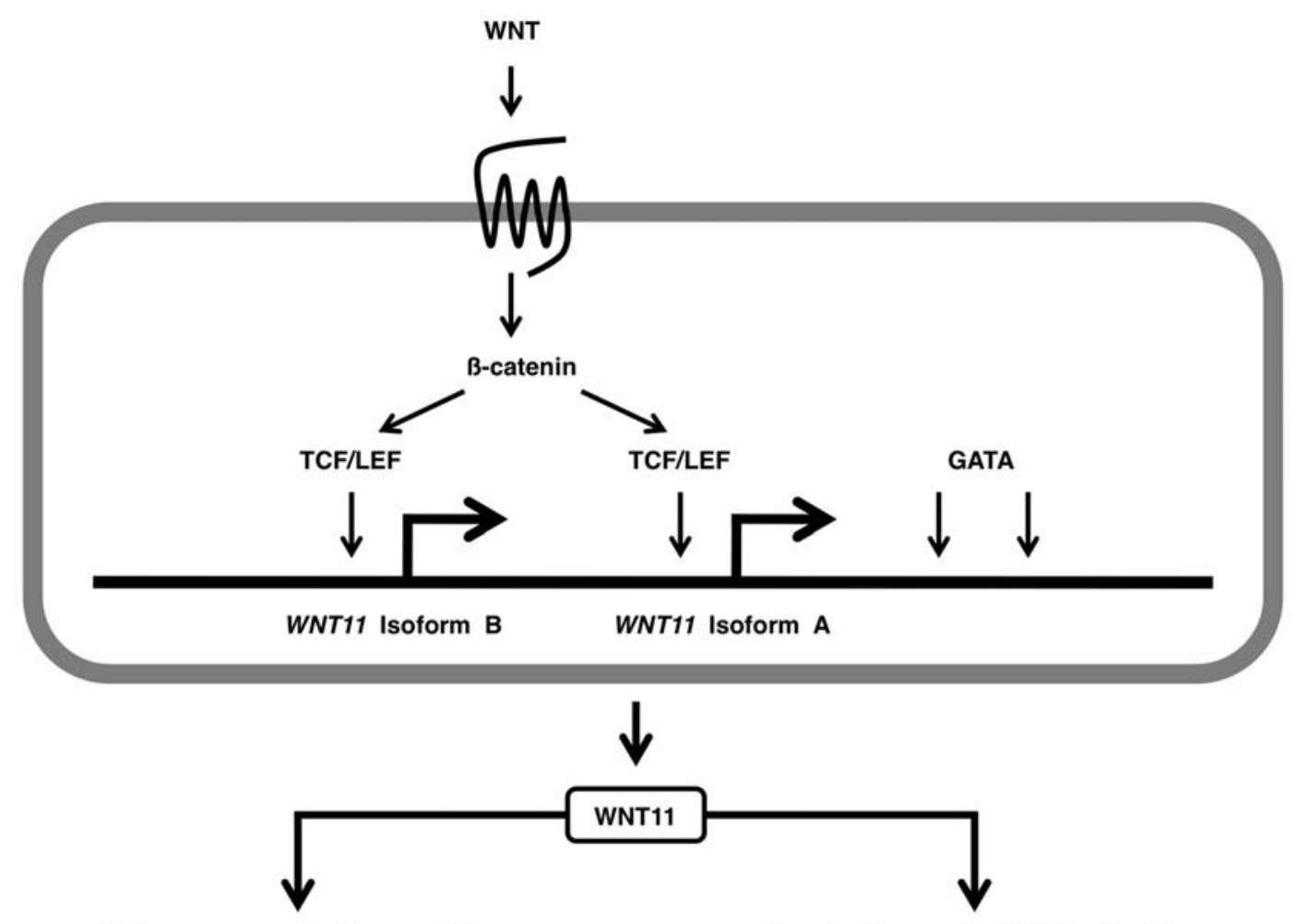

Cell movement during evolution

Break of canonical WNT signaling

Tumor invasion during carcinogenesis

Figure 2. Canonical WNT-to-WNT11 signaling loop. Canonical WNT signals directly induce WNT11 upregulation. WNT11 activates non-canonical WNT signaling cascades to induce cellular movement. WNT11 also activates the $\mathrm{Ca}^{2+}$-MAP3K7-NLK signaling cascade to break the canonical WNT signaling. Canonical WNT-to-WNT11 signaling loop is involved in cellular migration during embryogenesis, and tumor invasion during carcinogenesis.

the 5'-adjacent position of exon 2, and WNT11 intron 2 were well conserved in mammalian WNT11 orthologs (data not shown). However, the genome sequence around the WNT11 promoter B region was absent in the NW_001222304.1 chimpanzee genome draft sequence due to sequencing gap, and the genome sequence corresponding to a part of WNT11 intron 2 was absent in the NW_001100387.1 macaque genome draft sequence due to sequencing gap.

Conserved transcription factor-binding sites within WNT11 regulatory regions. Based on manual inspection, we identified TCF/LEF-binding sites within the promoter B region (-48-bp position from the TSS of human WNT11 isoform B), and also within the promoter A region (-43-bp position from the TSS of human WNT11 isoform A). We also found double GATAbinding sites within intron 2 of human WNT11 gene (+933-bp and +5001-bp positions from TSS of human WNT11 isoform A). Double TCF/LEF- and double GATA-binding sites within the regulatory regions of human WNT11 gene were conserved in other mammalian WNT11 orthologs (Fig. 1).

Canonical WNT signals involved in WNT11 upregulation. Lin et al reported that B-catenin is required for Wnt11 upregulation in cardiac progenitors based on the observation in conditional Ctnnbl knockout mice (58). Ueno et al reported that recombinant Wnt3a protein induces Wnt11 upregulation in embryoid bodies derived from mouse embryonic stem (ES) cells (42). Gros et al reported that electroporation of plasmid containing activated form of $\beta$-catenin cDNA in the lateral domain of newly formed somites induces Wnt11 upregulation in the lateral somites (59). Together these facts indicate that the canonical WNT signals are involved in WNT11 upregulation during embryogenesis; however, precise mechanism of WNT11 upregulation by the canonical WNT signals remained unclear.

In this study, we identified conserved TCF/LEF-binding sites within proximal promoter region of WNT11 orthologs (Fig. 1). Based on these facts, it was concluded that the canonical WNT signals directly upregulate WNT11 transcription (Fig. 2).

GATA family members involved in WNT11 upregulation. Afouda et al reported that gata4 and gata6 are involved in wnt11 upregulation during Xenopus cardiogenesis (60). Afouda et al suggested that wnt11 is a direct target of gata family members, because gata-induced wnt11 upregulation is resistant to cycloheximide treatment. However, precise mechanism of WNT11 upregulation by GATA family members remained unclear.

In this study, we identified conserved GATA-binding sites within intron 2 of WNT11 orthologs (Fig. 1). Based on these facts, it was concluded that GATA family members directly upregulate WNT11 transcription.

Other regulatory signaling cascades. WNT signaling cascades cross-talk with Hedgehog, Notch, FGF/RTK, and TGFß/ BMP signaling cascades (61-65). There are several reports that Hedgehog and Notch signaling cascades are involved in WNT11 upregulation (66-68). GLI and CSL are representative 
transcription factors involved in the regulation of Hedgehog (69-71) and Notch (72-74) target genes, respectively. However, conserved GLI- or CSL-binding site was not identified within the regulatory regions of mammalian WNT11 orthologs (data not shown). Hedgehog and Notch signaling cascades might be involved in WNT11 transcription through indirect mechanisms.

\section{Discussion}

Refined integrative genomic analyses of WNT11 were carried out to elucidate the mechanisms of WNT11 transcription in this study. The WNT11 gene at human chromosome 11q13.5 was found to encode two isoforms by using alternative first exons. WNT11 isoform A consists of exons 2, 3, 4, 5 and 6, whereas WNT11 isoform B consists of exons 1, 2, 3, 4, 5 and 6 (Fig. 1). Because the open reading frame spans exons 2-6, two WNT11 isoforms encode an identical WNT11 protein.

Canonical WNT signals and GATA family members are involved in WNT11 transcription during embryogenesis of model animals (42,58-60); however, precise mechanisms of WNT11 transcription remained unclear. TCF/LEF-binding site within the proximal promoter regions, and double GATAbinding sites within intron 2 of human WNT11 gene were identified in this study (Fig. 1). In addition, these TCF/LEFand GATA-binding sites within the regulatory regions of human WNT11 gene were conserved in other mammalian WNT11 orthologs (Fig. 1). Together these facts indicate that WNT11 transcription is directly upregulated by canonical WNT signals and GATA family members.

Canonical WNT signals induce WNT11 upregulation, and then WNT11 activates non-canonical WNT signaling cascades to induce cellular movement. WNT11 also activates the $\mathrm{Ca}^{2+}$ MAP3K7-NLK signaling cascade to attenuate the canonical WNT signaling. Canonical WNT-to-WNT11 signaling loop is involved in cellular migration during embryogenesis as well as tumor invasion during carcinogenesis (Fig. 2). The canonical WNT-to-WNT11 signaling loop is a potent target of cancer therapeutics, especially for the inhibition of invasion and metastasis.

\section{References}

1. Katoh M: Regulation of WNT signaling molecules by retinoic acid during neuronal differentiation in NT2 cells: Threshold model of WNT action. Int J Mol Med 10: 683-687, 2002.

2. Pandur P, Maurus D and Kühl M: Increasingly complex: new players enter the Wnt signaling network. Bioessays 24: 881-884, 2002.

3. Moon RT, Kohn AD, De Ferrari GV and Kaykas A: WNT and ß-catenin signalling: diseases and therapies. Nat Rev Genet 5: 691-701, 2004.

4. Gordon MD and Nusse R: Wnt signaling: multiple pathways, multiple receptors, and multiple transcription factors. J Biol Chem 281: 22429-22433, 2006.

5. Clevers H: Wnt/ß-catenin signaling in development and disease. Cell 127: 469-480, 2006.

6. Katoh M: WNT and FGF gene clusters. Int J Oncol 21: 1269-1273, 2002.

7. He X, Saint-Jeannet JP, Wang Y, Nathans J, Dawid I and Varmus H: A member of the Frizzled protein family mediating axis induction by Wnt5A. Science 275: 1652-1654, 1997.

8. Swain RK, Katoh M, Medina A and Steinbeisser H: Xenopus frizzled-4S, a splicing variant of Xfz4, is a context-dependent activator and inhibitor of Wnt/B-catenin signaling. Cell Commun Signal 3: 12, 2005.
9. Mikels AJ and Nusse R: Purified Wnt5a protein activates or inhibits B-catenin-TCF signaling depending on receptor context. PLoS Biol 4: e115, 2006.

10. Katoh M and Katoh M: WNT signaling pathway and stem cell signaling network. Clin Cancer Res 13: 4042-4045, 2007.

11. Katoh M: WNT/PCP signaling pathway and human cancer. Oncol Rep 14: 1583-1588, 2005.

12. Keeble TR, Halford MM, Seaman C, et al: Wnt receptor Ryk is required for Wnt5a-mediated axon guidance on contralateral side of corpus callosum. J Neurosci 26: 5840-5848, 2006.

13. Dejmek J, Säfholm A, Kamp Nielsen C, Andersson T and Leandersson $\mathrm{K}$ : Wnt $5 \mathrm{a} / \mathrm{Ca}^{2+}$-induced NFAT activity is counteracted by $\mathrm{Wnt} 5 \mathrm{a} / \mathrm{Yes}-\mathrm{Cdc} 42-\mathrm{CK} 1 \alpha$ signaling in human mammary epithelial cells. Mol Cell Biol 26: 6024-6036, 2006.

14. Zhang X, Zhu J, Yang GY, et al: Dishevelled promotes axon differentiation by regulating aPKC. Nat Cell Biol 9: 743-754, 2007.

15. Katoh M: Networking of WNT, FGF, Notch, BMP, and Hedgehog signaling pathways during carcinogenesis. Stem Cell Rev 3: 30-38, 2007.

16. Fukuda T, Chen L, Endo T, et al: Antisera induced by infusions of autologous Ad-CD154-leukemia B cells identify ROR1 as an oncofetal antigen and receptor for Wnt5a. Proc Natl Acad Sci USA 105: 3047-3052, 2008.

17. Katoh M: WNT signaling in stem cell biology and regenerative medicine. Curr Drug Targets 9: 565-570, 2008.

18. Lako M, Strachan T, Bullen P, et al: Isolation, characterisation and embryonic expression of WNT11. Gene 219: 101-110, 1998.

19. Kirikoshi H, Sekihara H and Katoh M: Molecular cloning and characterization of human WNT11. Int J Mol Med 8: 651-656, 2001.

20. Adamson MC, Dennis C, Delaney S, et al: Isolation and genetic mapping of murine Wnt11 and Wnt12. Genomics 24: 9-13, 1994.

21. Eisenberg CA, Gourdie RG and Eisenberg LM: Wntl1 is expressed in early avian mesoderm and required for the differentiation of the quail mesoderm cell line QCE-6. Development 124: 525-536, 1997.

22. Ku M and Melton DA: Xwnt11: a maternally expressed Xenopus wnt gene. Development 119: 1161-1173, 1993.

23. Heisenberg CP, Tada M, Rauch GJ, et al: Silberblick/Wnt11 mediates convergent extension movements during zebrafish gastrulation. Nature 405: 76-81, 2000.

24. Matsui T, Raya A, Kawakami Y, et al: Noncanonical Wnt signaling regulates midline convergence of organ primordial during zebrafish development. Genes Dev 19: 164-175, 2005.

25. Garriock RJ, D'Agostino SL, Pilcher KC and Krieg PA: Wnt11-R, a protein closely related to mammalian Wnt11, is required for heart morphogenesis in Xenopus. Dev Biol 279: 179-192, 2005.

26. Matthews HK, Broders-Bondon F, Thiery JP and Mayor R: Wnt11r is required for cranial neural crest migration. Dev Dyn 237: 3404-3409, 2008

27. Kühl M, Sheldahl LC, Malbon CC and Moon RT: $\mathrm{Ca}^{2+}$ calmodulin-dependent protein kinase II is stimulated by Wnt and Frizzled homologs and promotes ventral cell fates in Xenopus. J Biol Chem 275: 12701-12711, 2000 .

28. Djiane A, Riou J, Umbhauer M, Boucaut J and Shi D: Role of frizzled 7 in the regulation of convergent extension movements during gastrulation in Xenopus laevis. Development 127: 3091-3100, 2000.

29. Marlow F, Topczewski J, Sepich D and Solnica-Krezel L: Zebrafish Rho kinase 2 acts downstream of Wnt11 to mediate cell polarity and effective convergence and extension movements. Curr Biol 12: 876-884, 2002.

30. Penzo-Mendèz A, Umbhauer M, Djiane A, Boucaut JC and Riou JF: Activation of Gß $\gamma$ signaling downstream of Wnt-11/ Xfz7 regulates Cdc42 activity during Xenopus gastrulation. Dev Biol 257: 302-314, 2003.

31. Tao Q, Yokota C, Puck H, et al: Maternal wnt11 activates the canonical wnt signaling pathway required for axis formation in Xenopus embryos. Cell 120: 857-871, 2005.

32. Anton R, Kestler HA and Kühl M: B-catenin signaling contributes to stemness and regulates early differentiation in murine embryonic stem cells. FEBS Lett 581: 5247-5254, 2007.

33. Tahinci E, Thorne CA, Franklin JL, et al: Lrp6 is required for convergent extension during Xenopus gastrulation. Development 134: 4095-4106, 2007

34. Kim GH, Her JH and Han JK: Ryk cooperates with Frizzled 7 to promote Wnt11-mediated endocytosis and is essential for Xenopus laevis convergent extension movements. J Cell Biol 182: 1073-1082, 2008. 
35. Ulrich F, Concha ML, Heid PJ, et al: Slb/Wnt11 controls hypoblast cell migration and morphogenesis at the onset of zebrafish gastrulation. Development 130: 5375-5384, 2003.

36. Formstone CJ and Mason I: Combinatorial activity of Flamingo proteins directs convergence and extension within the early zebrafish embryo via the planar cell polarity pathway. Dev Biol 282: 320-335, 2005

37. Carron C, Bourdelas A, Li HY, Boucaut JC and Shi DL: Antagonistic interaction between IGF and Wnt/JNK signaling in convergent extension in Xenopus embryo. Mech Dev 122: 1234-1247, 2005.

38. De Calisto J, Araya C, Marchant L, Riaz CF and Mayor R: Essential role of non-canonical Wnt signalling in neural crest migration. Development 132: 2587-2597, 2005.

39. Eisenberg CA and Eisenberg LM: WNT11 promotes cardiac tissue formation of early mesoderm. Dev Dyn 216: 45-58, 1999.

40. Pandur P, Läsche M, Eisenberg LM and Kühl M: Wnt11 activation of a non-canonical Wnt signalling pathway is required for cardiogenesis. Nature 418: 636-641, 2002.

41. Koyanagi M, Haendeler J, Badorff C, et al: Non-canonical Wnt signaling enhances differentiation of human circulating progenitor cells to cardiomyogenic cells. J Biol Chem 280: 16838-16842, 2005.

42. Ueno S, Weidinger G, Osugi T, et al: Biphasic role for Wnt/ $\beta$-catenin signaling in cardiac specification in zebrafish and embryonic stem cells. Proc Natl Acad Sci USA 104: 9685-9690, 2007.

43. Flaherty MP and Dawn B: Noncanonical Wnt11 signaling and cardiomyogenic differentiation. Trends Cardiovasc Med 18: 260-268, 2008.

44. Kispert A, Vainio S, Shen L, Rowitch DH and McMahon AP: Proteoglycans are required for maintenance of Wnt-11 expression in the ureter tips. Development 122: 3627-3637, 1996.

45. Majumdar A, Vainio S, Kispert A, McMahon J and McMahon AP: Wnt11 and Ret/Gdnf pathways cooperate in regulating ureteric branching during metanephric kidney development. Development 130: 3175-3185, 2003.

46. Bergwitz C, Wendlandt T, Kispert A and Brabant G: Wnts differentially regulate colony growth and differentiation of chondrogenic rat calvaria cells. Biochim Biophys Acta 1538: 129-140, 2001.

47. Sekiya I, Vuoristo JT, Larson BL and Prockop DJ: In vitro cartilage formation by human adult stem cells from bone marrow stroma defines the sequence of cellular and molecular events during chondrogenesis. Proc Natl Acad Sci USA 99: 4397-4402, 2002 .

48. Wai DH, Schaefer KL, Schramm A, et al: Expression analysis of pediatric solid tumor cell lines using oligonucleotide microarrays. Int J Oncol 20: 441-451, 2002.

49. Üren A, Wolf V, Sun YF, et al: Wnt/Frizzled signaling in Ewing sarcoma. Pediatr Blood Cancer 43: 243-249, 2004.

50. Zhu H, Mazor M, Kawano Y, et al: Analysis of Wnt gene expression in prostate cancer: mutual inhibition by WNT11 and the androgen receptor. Cancer Res 64: 7918-7926, 2004.

51. Katoh Y and Katoh M: Conserved POU-binding site linked to SP1-binding site within FZD5 promoter. Int J Oncol 30: 751-755, 2007.

52. Katoh $\mathrm{M}$ and Katoh M: Comparative integromics on FZD7 orthologs. Int J Mol Med 19: 529-533, 2007.

53. Katoh Y and Katoh M: Comparative genomics on PROM1 gene encoding stem cell marker CD133. Int J Mol Med 19: 967-970, 2007.
54. Katoh M and Katoh M: Conserved POU/OCT- and GATAbinding sites in 5'-flanking promoter region of mammalian $W N T 8 B$ orthologs. Int J Oncol 30: 1273-1277, 2007.

55. Katoh $\mathrm{M}$ and Katoh $\mathrm{M}$ : Comparative integromics on noncanonical WNT or planar cell polarity signaling molecules. Int J Mol Med 20: 405-409, 2007.

56. Katoh $Y$ and Katoh Y: Integrative genomic analyses on GLI2. Int J Oncol 33: 881-886, 2008.

57. Katoh Y and Katoh M: Comparative genomics on WNT11 gene. Int J Mol Med 15: 879-883, 2005.

58. Lin L, Cui L, Zhou W, et al: B-catenin directly regulates Islet1 expression in cardiovascular progenitors and is required for multiple aspects of cardiogenesis. Proc Natl Acad Sci USA 104: 9313-9318, 2007.

59. Gros J, Serralbo O and Marcelle C: WNT11 acts as a directional cue to organize the elongation of early muscle fibres. Nature 457: 589-593, 2009.

60. Afouda BA, Martin J, Liu F, et al: GATA transcription factors integrate Wnt signalling during heart development. Development 135: 3185-3190, 2008.

61. Nusse R: Wnts and Hedgehogs: lipid-modified proteins and similarities in signaling mechanisms at the cell surface. Development 130: 5297-5305, 2003

62. Beachy PA, Karhadkar SS and Berman DM: Tissue repair and stem cell renewal in carcinogenesis. Nature 432: 324-331, 2004.

63. Garciadiego-Cazares D, Rosales C, Katoh M and ChimalMonroy J: Coordination of chondrocyte differentiation and joint formation by $\alpha 5 \beta 1$ integrin in the developing appendicular skeleton. Development 131: 4735-4742, 2004.

64. Katoh M: Dysregulation of stem cell signaling network due to germline mutation, SNP, Helicobacter pylori infection, epigenetic change, and genetic alteration in gastric cancer. Cancer Biol Ther 6: 832-839, 2007.

65. Katoh Y and Katoh M: Hedgehog signaling, epithelial-tomesenchymal transition, and miRNA. Int J Mol Med 22: 271-275, 2008.

66. Marcelle C, Stark MR and Bronner-Fraser M: Coordinate actions of BMPs, Wnts, Shh and noggin mediate patterning of the dorsal somite. Development 124: 3955-3963, 1997.

67. Mullor JL, Dahmane N, Sun T and Ruiz i Altaba A: Wnt signals are targets and mediators of Gli function. Curr Biol 11: 769-773, 2001

68. Koyanagi M, Bushoven P, Iwasaki M, et al: Notch signaling contributes to the expression of cardiac markers in human circulating progenitor cells. Circ Res 101: 1139-1145, 2007.

69. Lum L and Beachy PA: The Hedgehog response network: sensors, switches, and routes. Science 304: 1755-1759, 2004

70. Hooper JF and Scott MP: Communicating with Hedgehogs. Nat Rev Mol Cell Biol 6: 306-317, 2005.

71. Katoh Y and Katoh M: Hedgehog signaling in gastric cancer. Cancer Biol Ther 4: 1050-1054, 2005.

72. Artavanis-Tsakonas S, Rand MD and Lake RJ: Notch signaling: cell fate control and signal integration in development. Science 284: 770-776, 1999.

73. Radtke F and Raj K: The role of Notch in tumorigenesis: oncogene or tumor suppressor? Nat Rev Cancer 3: 765-767, 2003

74. Katoh $\mathrm{M}$ and Katoh M: Notch signaling in gastrointestinal tract. Int J Oncol 30: 247-251, 2007. 